Recherches en didactique des langues et des cultures

Les cahiers de l'Acedle

13-3 | 2016

Travailler sur et à partir de textes écrits en classe de langue étrangère

\title{
Compréhension à la lecture et expression écrite en langue étrangère dans l'enseignement secondaire
}

Christiane Blondin, Christelle Goffin et Ariane Baye

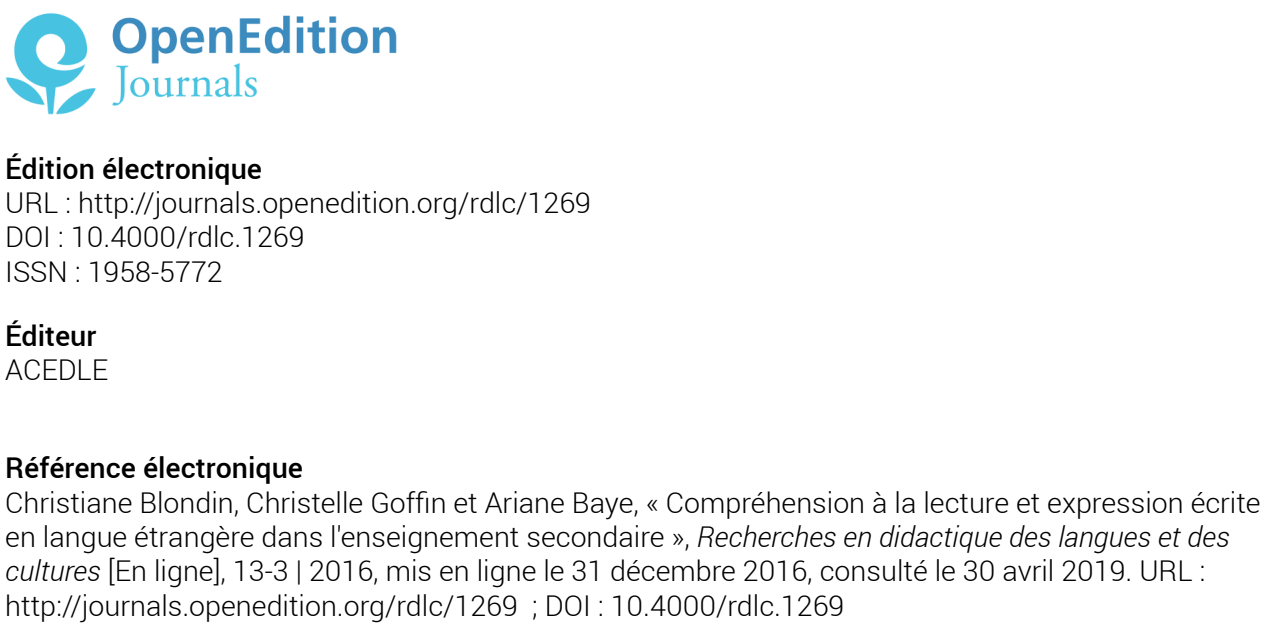

Ce document a été généré automatiquement le 30 avril 2019

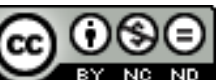

Recherches en didactique des langues et des cultures is licensed under a Creative Commons AttributionNonCommercial-NoDerivatives 4.0 International License 


\title{
Compréhension à la lecture et expression écrite en langue étrangère dans l'enseignement secondaire
}

\author{
Christiane Blondin, Christelle Goffin et Ariane Baye
}

\section{L'étude européenne' ${ }^{1}$ des compétences ${ }^{2}$ en langues}

1 Depuis de nombreuses années, l'évaluation des compétences en langues modernes sur un plan international fait défaut (Blondin \& Demeuse, 2000). Après l'enquête de $l^{\prime}$ International Association for the Evaluation of Educational Achievement (IEA) portant sur les performances en anglais menée en 1972, les autres tentatives de l'IEA ou de l'Organisation de Coopération et de Développement Économiques (Ocdé) dans le domaine se sont heurtées à la difficulté de concevoir une évaluation globale des performances en langues étrangères, ainsi qu'à la difficulté de prendre en compte l'éventail des langues possibles et les différents curriculums en termes d'âge du début de l'apprentissage et de nombre de périodes hebdomadaires (Lafontaine \& Blondin, 2004). Or, dans le contexte de la globalisation, mais aussi dans le cadre du projet européen, des informations sur la capacité des systèmes éducatifs à former des citoyens multilingues sont cruciales. En plus des données recueillies par Eurydice sur l'enseignement des langues en Europe (EACEA/ Eurydice, 2012), l'Union européenne a donc décidé en 2005 de mettre au point un indicateur des compétences linguistiques en vue de fournir aux états membres une base comparative sur laquelle fonder leurs choix stratégiques.

2 C'est dans ce but que la Commission européenne a organisé une évaluation à grande échelle des compétences en langues, pilotée par un Conseil consultatif composé de représentants des pays. Un consortium de centres de recherche appelé SurveyLang (Surveylang, 2012), constitué à cet effet, a été chargé d'assurer la gestion et la 
coordination internationales de la première Étude européenne des compétences en langues ou ESLC (en anglais, European Survey on Language Competencies).

3 À l'instar des autres surveys internationaux portant sur les rendements scolaires, SurveyLang est une étude essentiellement descriptive, permettant de dresser le profil de différents systèmes éducatifs à un moment donné sur des compétences jugées importantes. Les forces et les faiblesses peuvent être liées à des décisions politiques en termes structurels, en termes de choix quantitatifs et qualitatifs effectués pour établir le curriculum dans le domaine des langues étrangères, comme le font Araùjo et Dinis da Costa (2013) à partir des données de SurveyLang. Les conclusions sont cependant basées sur des analyses corrélationnelles, qui doivent idéalement être croisées avec les résultats d'autres études, et notamment de la recherche expérimentale sur les caractéristiques des programmes et des enseignants efficaces en termes d'apprentissage des langues étrangères (voir à ce sujet Dixon et al., 2012).

4 Après la présentation du dispositif d'évaluation, la contribution sera centrée sur les performances des élèves en lecture et expression écrite. Les performances des élèves seront ensuite mises en lien avec les contextes d'apprentissage des langues décrits par les professeurs en réponse à un questionnaire qui porte notamment sur leurs pratiques pédagogiques et les aspects qu'ils jugent prioritaires pour l'apprentissage d'une langue. Ces informations dépassent le cadre des deux domaines d'évaluation visés ici, dans le but de mieux appréhender le contexte de l'apprentissage des langues étrangères.

\section{Le dispositif}

5 L'ESLC s'appuie largement sur les acquis du Programme international pour le suivi des acquis des élèves (PISA) de l'Ocde et s'est fixé des standards de qualité élevés pour les différentes facettes de l'étude, de la fidélité des traductions à la rigueur des procédures d'administration des épreuves.

6 L'évaluation a porté sur les compétences en compréhension à la lecture et à l'audition et en production écrite des élèves de dernière année du secondaire inférieur (CITE 2, selon la Classification internationale type de l'éducation - UNESCO, 1997) dans les deux langues les plus enseignées dans chaque pays. Cependant lorsque les élèves du CITE 2 ne remplissaient pas une condition de durée minimum d'apprentissage de la langue cible (avoir étudié la langue cible depuis au moins une année scolaire complète), l'évaluation a porté sur la deuxième année du secondaire supérieur (CITE 3).

7 Lors de la première édition de l'ESLC en $2011^{3}$, qui fait l'objet du présent article, les épreuves étaient disponibles dans les cinq langues les plus enseignées dans l'Union européenne, soit l'anglais, l'allemand, le français, l'italien et l'espagnol.

8 Un test d'orientation ${ }^{4}$ a été administré et corrigé au préalable, de façon à prendre en compte les différences entre les compétences individuelles et à confronter chaque élève à une épreuve d'un niveau de difficulté adapté (bien entendu, les résultats de tous les élèves testés ont ensuite été replacés sur une même échelle grâce à une procédure statistique basée sur des items communs aux différentes épreuves).

Dans chaque établissement d'enseignement secondaire sélectionné, le directeur, les professeurs enseignant la langue cible au niveau CITE concerné et les élèves de l'échantillon ont complété des questionnaires de contexte. Ces questionnaires permettent de décrire les conditions dans lesquelles les élèves apprennent la langue cible (formation 
continuée des professeurs, moyens dont ils disposent, avis des élèves sur les langues étrangères, etc.) et, dans certains cas, de mettre en évidence des facteurs favorables à cet apprentissage (voir le rapport technique de l'étude sur le site: http://ec.europa.eu/ languages/policy/strategic-framework/documents/language-survey-technicalreport_en.pdf).

10 À l'exception de l'épreuve d'expression écrite, toujours réalisée "à la main", les tests et le questionnaire destinés aux élèves ont été administrés soit sur ordinateur, soit dans une version papier/crayon.

\section{Le Cadre européen commun de référence pour les langues}

11 Les tests ont été élaborés dans chacune des langues en fonction de la grille des niveaux de compétence proposée dans le Cadre européen commun de référence pour les langues ou CECRL (Conseil de l'Europe, 2001).

Quatre niveaux du CECRL ont été retenus: les niveaux A1 et A2 correspondant à un utilisateur élémentaire et les niveaux B1 et B2 correspondant à un utilisateur indépendant (ou autonome). Les élèves capables d'atteindre les niveaux $\mathrm{C} 1$ ou $\mathrm{C} 2$ se sont vu attribuer le code B2. À l'autre extrémité de l'échelle, un niveau Pré-A1 a été créé.

\section{La rédaction des items}

13 Les items ont été construits par les experts des différentes langues associés dans le consortium SurveyLang, sur la base des niveaux du CECRL et de la spécification de tâches.

14 La figure 1 ci-après présente un exemple de tâche pour l'allemand. Chaque tâche est définie par différentes caractéristiques. Ainsi, la tâche présentée ci-dessous est caractérisée par les éléments suivants :

- sa cible : trouver une information factuelle prévisible;

- le type de texte : dans une annonce ;

- le type de question: question à choix multiple, choix entre trois options sur la base d'un texte court centré sur l'information.

La réussite de $60 \%$ des items de cette tâche correspond au niveau A1 et de $100 \%$ des items au niveau $\mathrm{A} 2$. 
Figure 1. Exemple de tâche de compréhension à la lecture en allemand.

Du liest eine Anzeige. Sophie sucht ihre Katze. Wähle bei den folgenden 4 Aufgaben die richtige Lösung $A, B$ oder $C$.

Wer hat Leonie gesehen?

Leonie ist meine kleine Katze. Sie ist weiß mit schwarzen Punkten. Sie ist noch klein und sehr süß, hat braune Augen und trägt ein graues Halsband. Sie geht gern auf den

Häusern zwischen der Bäckerei Hansmann und der Plauenerstraße spazieren. Seit

Montag ist sie weg und heute ist schon Donnerstag. Für eine kleine Katze ist das schon eine lange Zeit!

Haben Sie Leonie in Ihrem Garten oder unter Ihrem Auto gesehen? Dann rufen Sie mich bitte an! Achtung: Leonie darf man nicht tragen und sie trinkt keine Milch.

Vielen Dank für Ihre Hilfe!

Sophie Mann 08808484349

1 Welche Farbe hat Leonie?
A schwarz und grau
B braun und grau
C schwarz und weiß

2 Wo ist Leonie gern?
A in Gärten
B unter Autos
C auf Häusern

3 Wie lange ist Leonie schon weg?
A einen Tag
B mehrere Tage
C eine Woche

4 Ich finde Leonie. Ich soll ...
A zur Bäckerei gehen.
B Leonie Milch geben.
C Sophie anrufen.

16 Les consignes ont été données dans la langue cible. Pour réduire l'impact sur les performances d'un manque de familiarité avec les consignes en langue cible, des questions différentes de celles de l'enquête, mais de structure similaire, ont été proposées aux écoles : les professeurs étaient invités à les utiliser pour familiariser leurs élèves avec le mode d'évaluation.

17 Seules des questions à choix multiple ont été proposées pour la compréhension à la lecture et à l'audition, de façon à permettre une correction automatique et fiable. Alors que l'objet de la question se veut cohérent avec une approche fonctionnelle, son utilisation dans une enquête à grande échelle impose une forme qui l'éloigne de l'authenticité et lui donne une allure scolaire.

\section{L'équivalence des épreuves dans les différentes langues}

Comment les concepteurs des tests ont-ils tenté d'assurer la comparabilité des résultats entre les langues?

Contrairement à ce qui se passe habituellement dans les enquêtes internationales, l'option choisie a été de créer des tests parallèles dans les différentes langues d'évaluation au lieu de produire les items dans une langue, puis de les traduire. Ce choix limite, dans une certaine mesure, la comparabilité des résultats.

Des compétences évaluables ont été définies en cohérence avec le modèle sociocognitif de l'apprentissage des langues sur lequel se fonde le CECRL. Elles ont été associées à des types de tâches spécifiques, définis sur la base de l'expérience des divers centres associés dans le consortium. Lors de la rédaction des items, un processus systématique de validation inter-langues des tâches par les experts a permis de s'assurer que les constructs 
et la façon dont les tâches devaient les mesurer étaient bien compris de la même manière par tous.

21 Sur la base de catégories proposées par le CECRL, quatre "secteurs" ont été distingués (personnel, public, lié à l'enseignement et professionnel), chacun d'entre eux étant divisé en thèmes et sous-thèmes. Ainsi, par exemple, le secteur "personnel" comporte les thèmes famille, amis, loisirs, maison, objets et animaux familiers et le thème "loisirs" inclut les sous-thèmes sports, musique, cinéma, Internet, lecture, sorties. La campagne principale a utilisé 635 items regroupés en 143 tâches.

22 Par la suite, des experts désignés par les pays ont travaillé à établir l'équivalence des limites entre les niveaux dans les différentes langues cibles en prenant également en compte les résultats de la campagne principale de l'enquête.

23 Ainsi construites, les épreuves administrées aux élèves permettent de répartir ceux-ci entre les différents niveaux du CECRL et d'obtenir des pourcentages comparables pour les différentes langues et compétences testées.

\section{Le codage des épreuves de production écrite} production de l'élève correspond à la tâche assignée, c'est-à-dire la qualité de la communication de la matière ou des informations requises (points traités, développements adéquats, style approprié à la situation) ; d'autre part, l'adéquation de la production à la tâche prescrite en ce qui concerne la cohérence, le vocabulaire utilisé, l'organisation du message et son exactitude. Le premier aspect sera appelé "efficacité de la communication" et le second "correction de la langue cible". Les codeurs ont bénéficié d'une formation et leurs premiers codages ont fait l'objet d'un contrôle.

Pour chacun des critères de correction, la production de l'élève reçoit un code qui indique la zone de compétence dans laquelle elle se situe, sur une échelle de 1 à 3 (moins bien, à peu près aussi bien ou mieux que la copie étalon unique) ou de 1 à 5 , lorsque la référence se compose de deux productions d'élèves. Une partie des productions ont été soumises à un double codage. Enfin un échantillon des productions déjà codées deux fois localement a été envoyé au consortium où un spécialiste a attribué ses propres codes. Sur ces bases, SurveyLang a pu, dans certains cas, ajuster les codes attribués par les experts nationaux (par exemple s'il apparaissait que les experts d'un pays étaient systématiquement plus sévères que la référence).

\section{La participation}

27 Seize entités adjudiquées (pays ou structures subnationales telles que les Communautés belges, dont les systèmes éducatifs sont distincts) ont participé au premier cycle de l'ESLC (voir le tableau 1).

28 L'âge théorique le plus fréquent est 15 ans (16 populations cibles), suivi par 14 ans (10 populations cibles). Les Communautés flamande et germanophone de Belgique 
constituent des exceptions en testant des élèves particulièrement jeunes pour la première langue cible (13 ans d'âge théorique). La Bulgarie teste quant à elle des élèves particulièrement âgés (16 ans), pour les deux langues cibles.

Tableau 1 - Langues cibles retenues, âge théorique des élèves et année d'études fréquentée pour les entités adjudiquées participant à l'ESLC

\begin{tabular}{|c|c|c|c|c|c|c|}
\hline \multirow{2}{*}{ Entité adjudiquée } & \multicolumn{3}{|c|}{ Langue cible 1} & \multicolumn{3}{|c|}{ Langue cible 2} \\
\hline & $\begin{array}{l}\text { Langue } \\
\text { évaluée }\end{array}$ & Âge & $\begin{array}{l}\text { Année } \\
\text { d'études }\end{array}$ & $\begin{array}{l}\text { Langue } \\
\text { évaluée }\end{array}$ & Âge & $\begin{array}{l}\text { Année } \\
\text { d'études }\end{array}$ \\
\hline Angleterre & Français & 15 & 11 & Allemand & 15 & 11 \\
\hline Bulgarie & Anglais & 16 & 10 & Allemand & 16 & 10 \\
\hline Communauté flamande & Français & 13 & 8 & Anglais & 15 & 10 \\
\hline Communauté française & Anglais & 15 & 10 & Allemand & 15 & 10 \\
\hline $\begin{array}{l}\text { Communauté } \\
\text { germanophone }\end{array}$ & Français & 13 & 8 & Anglais & 15 & 10 \\
\hline Croatie & Anglais & 14 & 8 & Allemand & 14 & 8 \\
\hline Espagne & Anglais & 15 & 10 & Français & 15 & 10 \\
\hline Estonie & Anglais & 15 & 9 & Allemand & 15 & 9 \\
\hline France & Anglais & 14 & 9 & Espagnol & 14 & 9 \\
\hline Grèce & Anglais & 14 & 9 & Français & 14 & 9 \\
\hline Malte & Anglais & 15 & 11 & Italien & 15 & 11 \\
\hline Pays-Bas & Anglais & $14-15$ & $9-10$ & Allemand & $14-15$ & $9-10$ \\
\hline Pologne & Anglais & 15 & 9 & Allemand & 15 & 9 \\
\hline Portugal & Anglais & 14 & 9 & Français & 14 & 9 \\
\hline Slovénie & Anglais & 14 & 9 & Allemand & 14 & 9 \\
\hline Suède & Anglais & 15 & 9 & Espagnol & 15 & 9 \\
\hline
\end{tabular}

À côté de la variété en termes d'âge et d'année d'études, les élèves interrogés présentent également des profils diversifiés en termes de nombre d'années scolaires au cours desquelles ils ont reçu un enseignement dans la langue cible. Le passé scolaire de l'élève dans la langue cible est cependant difficile à quantifier. Une question porte sur le fait d'avoir eu des cours en langue cible dans les années scolaires antérieures, mais les possibilités de changements en termes de volume hebdomadaire et de choix de langue au fil du parcours scolaire sont parfois telles qu'il est difficile d'obtenir une information 
fiable à ce sujet. Cependant, des analyses secondaires menées par Araùjo et Dinis da Costa (2013) indiquent qu'un apprentissage précoce est souvent positivement lié à des taux de maitrise plus importants, ce que ne confirment pas nécessairement les études où le nombre d'heures d'enseignement peut être contrôlé (Dixon et al., 2012).

\section{Les performances des élèves en compréhension à la lecture et en production écrite} compréhension à la lecture et en expression écrite, par langue cible ${ }^{5}$, sont présentés dans les figures 2 à 4 .

Dans le cas de l'allemand (voir la figure 2), les profils établis sur la base des résultats obtenus dans les huit systèmes éducatifs où cette langue a été évaluée présentent un mode $^{6}(42 \%)$ en A1 pour les deux compétences.

Figure 2 - Pourcentages d'élèves atteignant les différents niveaux du CECRL en compréhension à la lecture et en expression écrite en allemand (avec les intervalles de confiance)

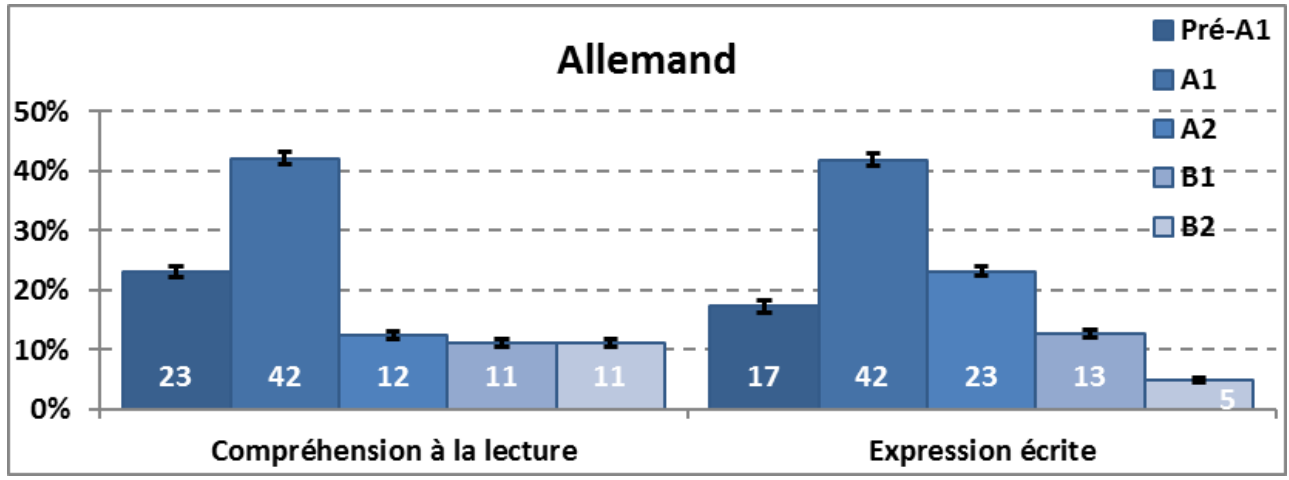

En compréhension à la lecture, près d'un quart des élèves (23\%) sont à la traine, puisqu'ils n'atteignent même pas le niveau A1; la proportion d'élèves en grande difficulté est un peu plus faible en expression écrite (17\%). Si les élèves plus avancés dans leur apprentissage de la lecture se répartissent également entre les trois niveaux supérieurs (11 ou $12 \%$ en A2, B1 ou B2), en expression écrite, plus le niveau s'élève, plus le pourcentage d'élèves qui l'atteignent est faible : on passe de $23 \%$ en A2 à $13 \%$ en B1 et seulement $5 \%$ en B2.

a situation est très différente dans le cas de l'anglais (voir la figure 3): en compréhension à la lecture, on observe une distribution bimodale, avec près du tiers des élèves (32\%) atteignant au moins le niveau B2. En outre, plus d'un quart des élèves ont atteint au moins le niveau A2 (12\% A2 et $15 \%$ B1). Les autres élèves sont cependant encore nombreux aux niveaux A1 (29 \%) et même Pré-A1 (13\%).

En expression écrite, la répartition des élèves entre les différents niveaux reflète une maitrise moins aboutie : beaucoup moins d'élèves ont atteint le niveau B2 (14\%), mais $33 \%$ se situent en B1 et un peu moins en A2 $(25 \%)$ et en A1 ( $21 \%)$. Huit pour cent seulement n'atteignent pas le niveau A1. 
Figure 3 - Pourcentages d'élèves atteignant les différents niveaux du CECRL en compréhension à la lecture et en expression écrite en anglais (avec les intervalles de confiance)

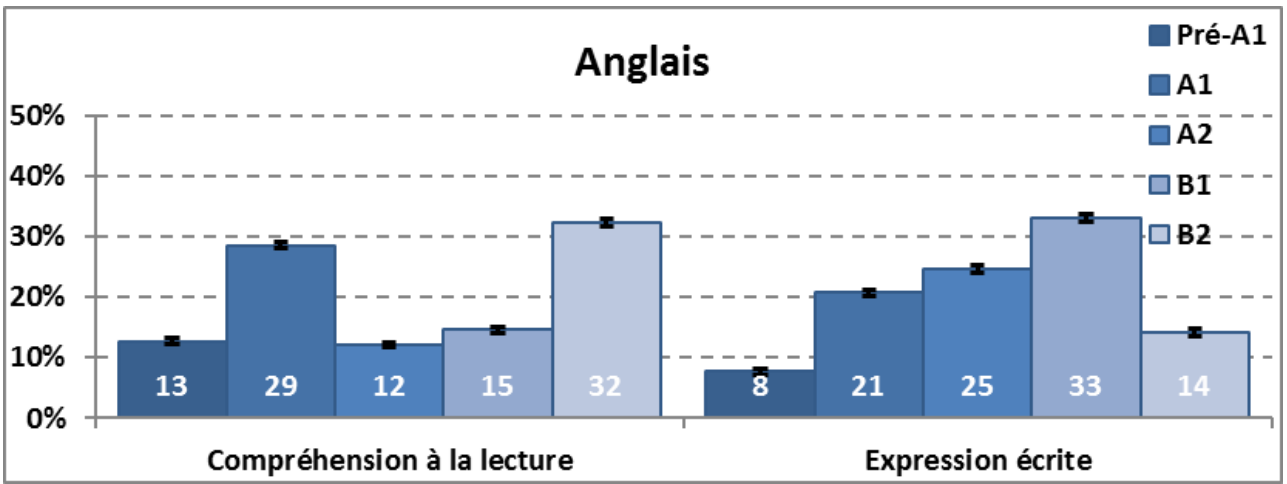

Lorsqu'il s'agit du français (voir la figure 4), le mode se situe en A1, pour les deux compétences, avec $45 \%$ des élèves en compréhension à la lecture et $35 \%$ en expression écrite. Un quart des élèves n'atteignent pas ce niveau en expression écrite (25\%) (ils sont un peu moins nombreux dans ce cas en compréhension à la lecture - $17 \%$ ). Pour les deux compétences, les pourcentages d'élèves tendent à diminuer avec l'augmentation des niveaux à partir de A1, mais la décroissance est plus forte dans le cas de l'expression écrite.

Figure 4 - Pourcentages d'élèves atteignant les différents niveaux du CECRL en compréhension à la lecture et en expression écrite en français (avec les intervalles de confiance)

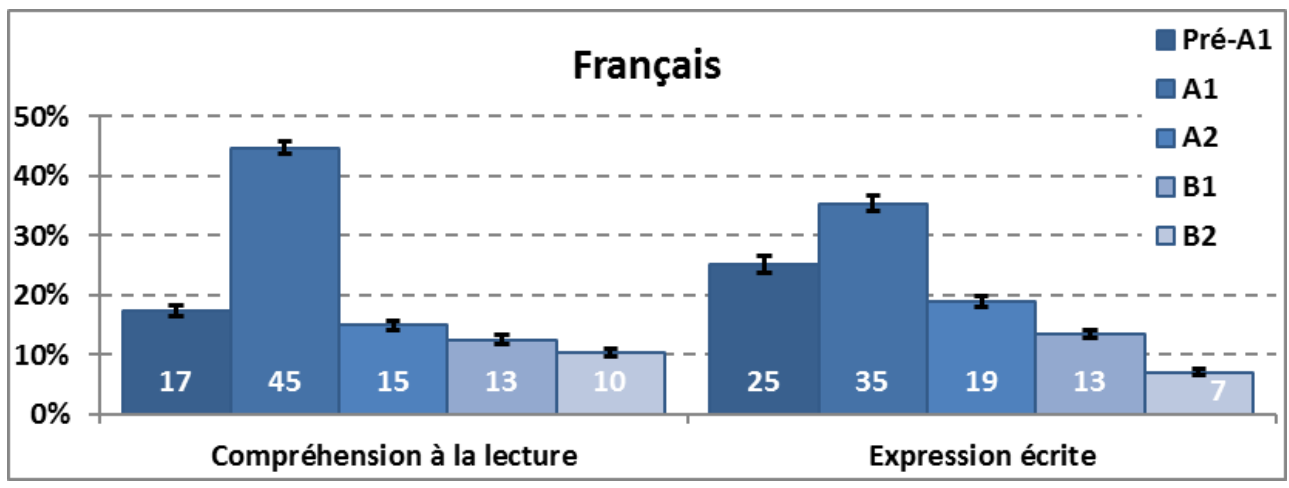

Les conditions de réalisation de l'enquête imposent une grande prudence quant à la comparaison des résultats relatifs aux différentes langues cibles. D'une part, en effet, les systèmes éducatifs concernés par chaque langue sont différents (15 pour l'anglais, mais seulement 8 pour l'allemand et 6 pour le français). D'autre part l'anglais est la langue la plus enseignée dans 13 cas sur 15 , tandis que le français n'est dans cette position que dans 2 cas (les Communautés flamande et germanophone de Belgique) et l'allemand est toujours en seconde position. S'il s'agit bien ici de la fréquence de l'enseignement de chaque langue dans chaque pays ou communauté, cette priorité quantitative se concrétise souvent dans l'ordre d'acquisition pour les élèves et en outre dans un nombre d'années d'étude différent : l'apprentissage scolaire de la langue la plus enseignée débute souvent plus tôt. Quoi qu'il en soit, on peut affirmer que les compétences en compréhension à la lecture et en expression écrite sont mieux maitrisées en anglais que dans les deux autres langues cibles analysées, et que les performances des élèves en allemand et en français partagent des points communs et correspondent davantage à un apprentissage en cours, 
non encore abouti, chez la plupart d'entre eux. Ces résultats doivent être mis en lien avec le fait que l'anglais est la langue la plus fréquemment adoptée comme première langue étrangère. Elle est aussi la langue perçue comme la plus utile et la plus facile à apprendre par la majorité des élèves. Le degré d'exposition à l'anglais est également élevé dans les médias (EACEA/Eurydice, 2012 ; SurveyLang, 2012).

Les figures 5 et 6 permettent de comparer les résultats en allemand pour les deux compétences dans les huit systèmes éducatifs où cette langue a été testée. Les pays ont été rangés de gauche à droite par ordre de performances croissantes en compréhension à la lecture en considérant le pourcentage d'élèves atteignant les niveaux B1 et B2: ainsi, les élèves des Pays-Bas, dont les performances en compréhension de la lecture sont les meilleures, sont très peu nombreux aux niveaux les plus faibles (Pré-A1, A1) et les plus nombreux aux niveaux les plus élevés (B2, B1).

Figures 5 et 6 - Pourcentages d'élèves atteignant les différents niveaux du CECRL en compréhension à la lecture (fig. 5) et expression écrite (fig. 6) en allemand dans chaque système éducatif

\section{Compréhension à la lecture, allemand}

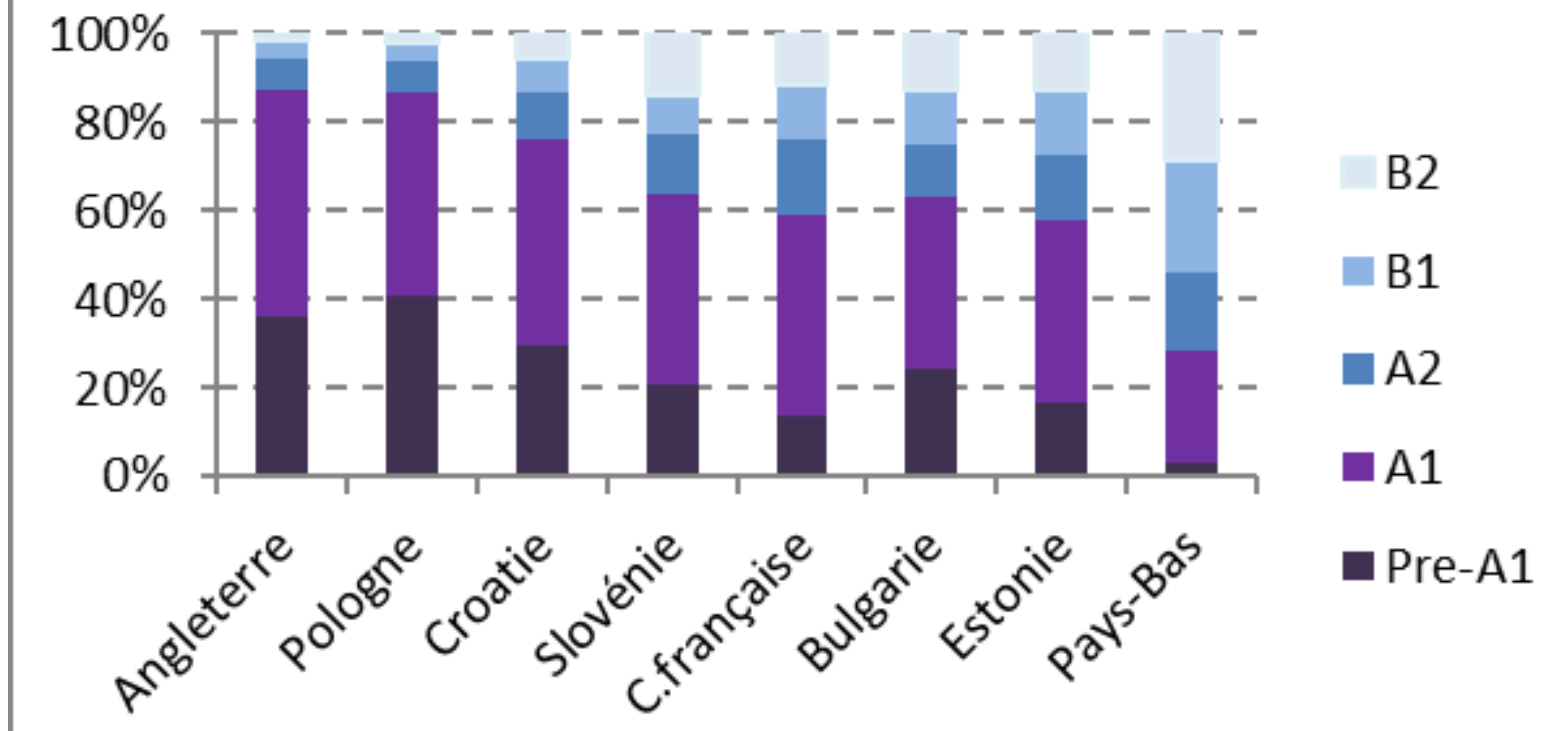




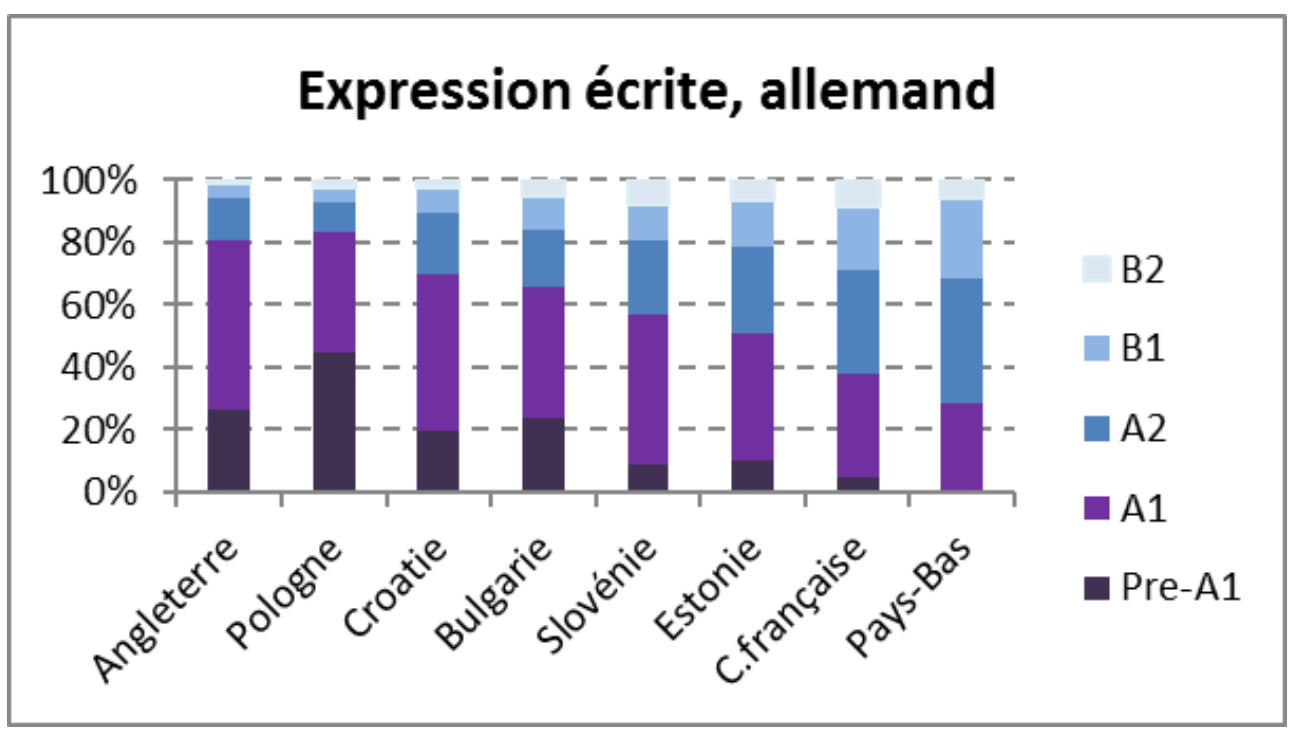

Avec $54 \%$ d'élèves considérés comme lecteurs indépendants en allemand, les Pays-Bas surclassent largement les autres pays participants. Environ un quart des élèves estoniens, bulgares, belges francophones et slovènes atteignent les niveaux B1 et B2. Dans les trois derniers pays, les pourcentages de lecteurs indépendants sont très faibles : $13 \%$ en Croatie, et seulement $6 \%$ en Pologne et en Angleterre.

En expression écrite, et bien que les Pays-Bas conservent la première place, on peut noter un net recul de ce pays en termes d'élèves atteignant les niveaux B1 et B2 (31\%). Par contre, le niveau d'autonomie est atteint par $29 \%$ des Belges francophones, qui sont donc un peu plus à l'aise en production écrite qu'en compréhension, ce qui est plutôt atypique. Pour le reste, le classement des pays en ce qui concerne l'expression écrite est globalement cohérent avec le classement en compréhension à la lecture.

Quinze systèmes éducatifs ont choisi d'évaluer leurs élèves en anglais. Les résultats sont présentés aux figures 7 et 8 .

Figures 7 et 8 - Pourcentages d'élèves atteignant les différents niveaux du CECRL en compréhension à la lecture (fig. 7) et expression écrite (fig. 8) en anglais dans chaque système éducatif

\section{Compréhension à la lecture, anglais}

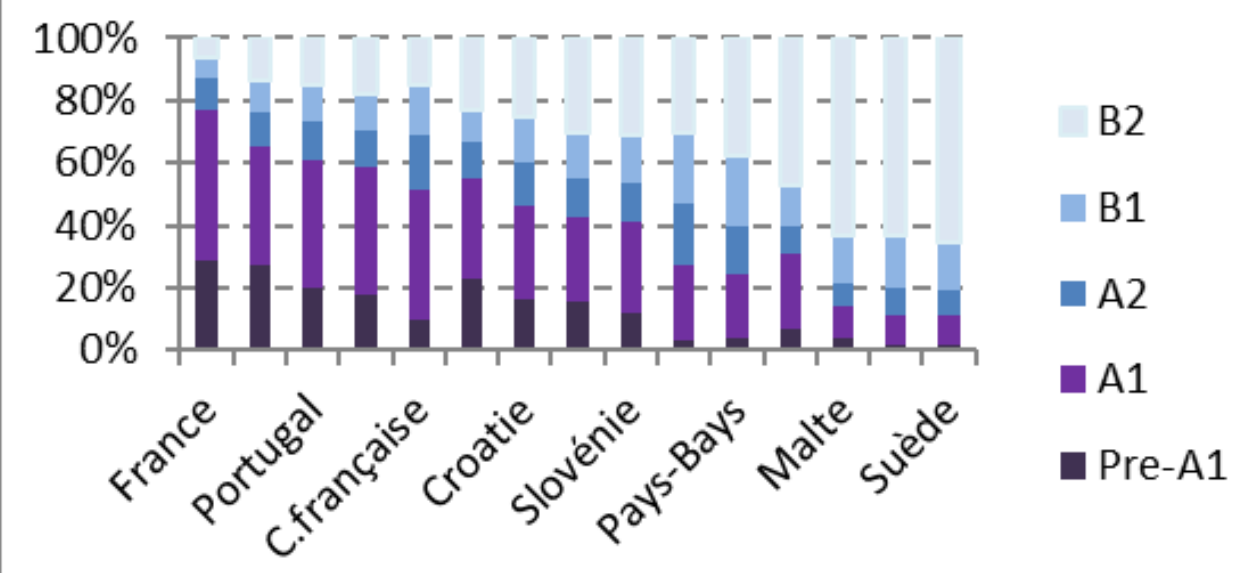




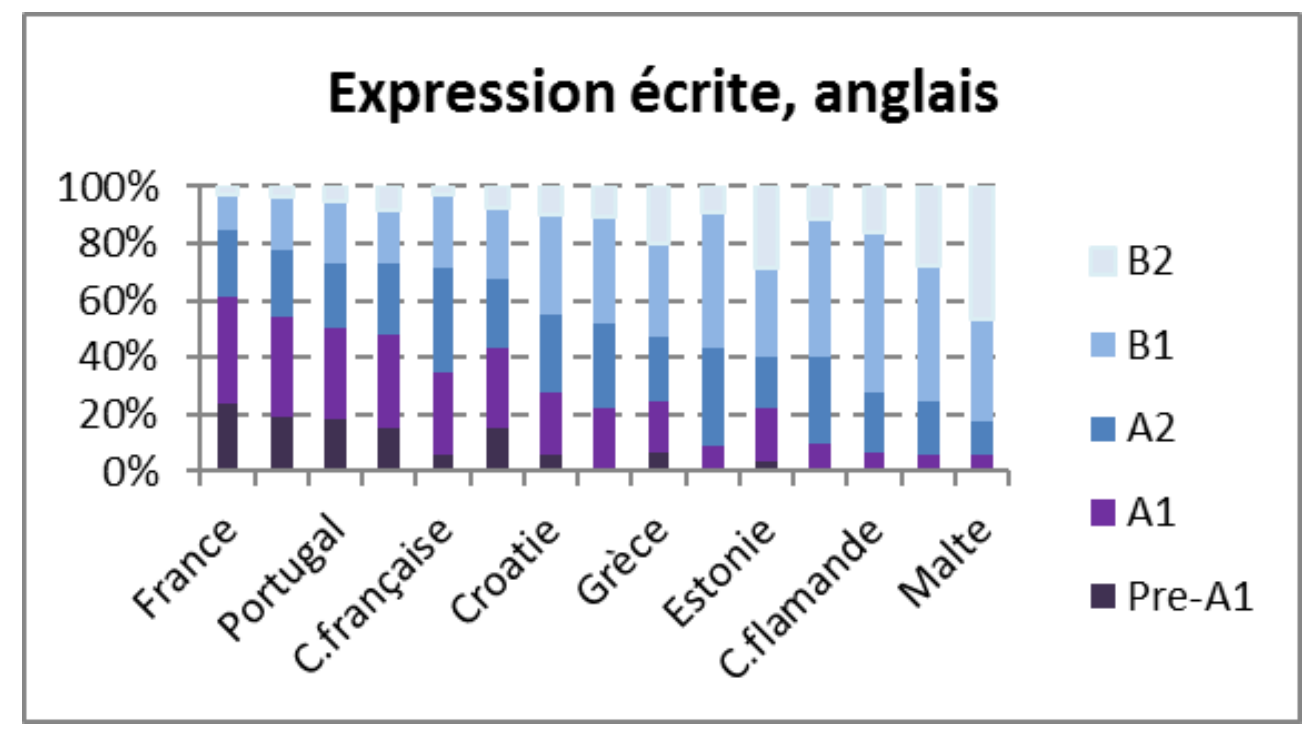

En Suède, en Communauté flamande et à Malte, les jeunes testés ont en général un excellent niveau de maitrise de la lecture en anglais, puisqu'environ $80 \%$ d'entre eux atteignent les niveaux B1 ou B2. Ces systèmes éducatifs s'illustrent également en expression écrite, même si, à la notable exception de Malte, les proportions de scripteurs indépendants sont plus faibles que celles de lecteurs indépendants en anglais. On note que les Pays-Bas et l'Estonie font partie, comme c'était le cas pour l'allemand, des pays qui se distinguent favorablement, avec environ $60 \%$ de lecteurs et de scripteurs indépendants en anglais. On note, comme c'était le cas pour l'allemand, une difficulté pour les élèves polonais à atteindre un niveau de maitrise suffisant. Les autres pays où les élèves sont le plus en difficulté en anglais sont les pays de langues romanes. En termes de constats plus généraux, on remarque non seulement que les positions relatives des pays sont assez proches quelle que soit la compétence envisagée, mais aussi que les proportions de lecteurs et de scripteurs indépendants sont très proches (à l'exception des systèmes évoqués ci-dessus et de la Grèce qui compte plus d'élèves à l'aise à l'écrit).

Le français a constitué une langue d'évaluation dans six systèmes éducatifs. Leurs résultats sont présentés dans les figures 9 et 10. 
Figures 9 et 10 - Pourcentages d'élèves atteignant les différents niveaux du CECRL en compréhension à la lecture (fig. 9) et expression écrite (fig. 10) en français dans chaque système éducatif
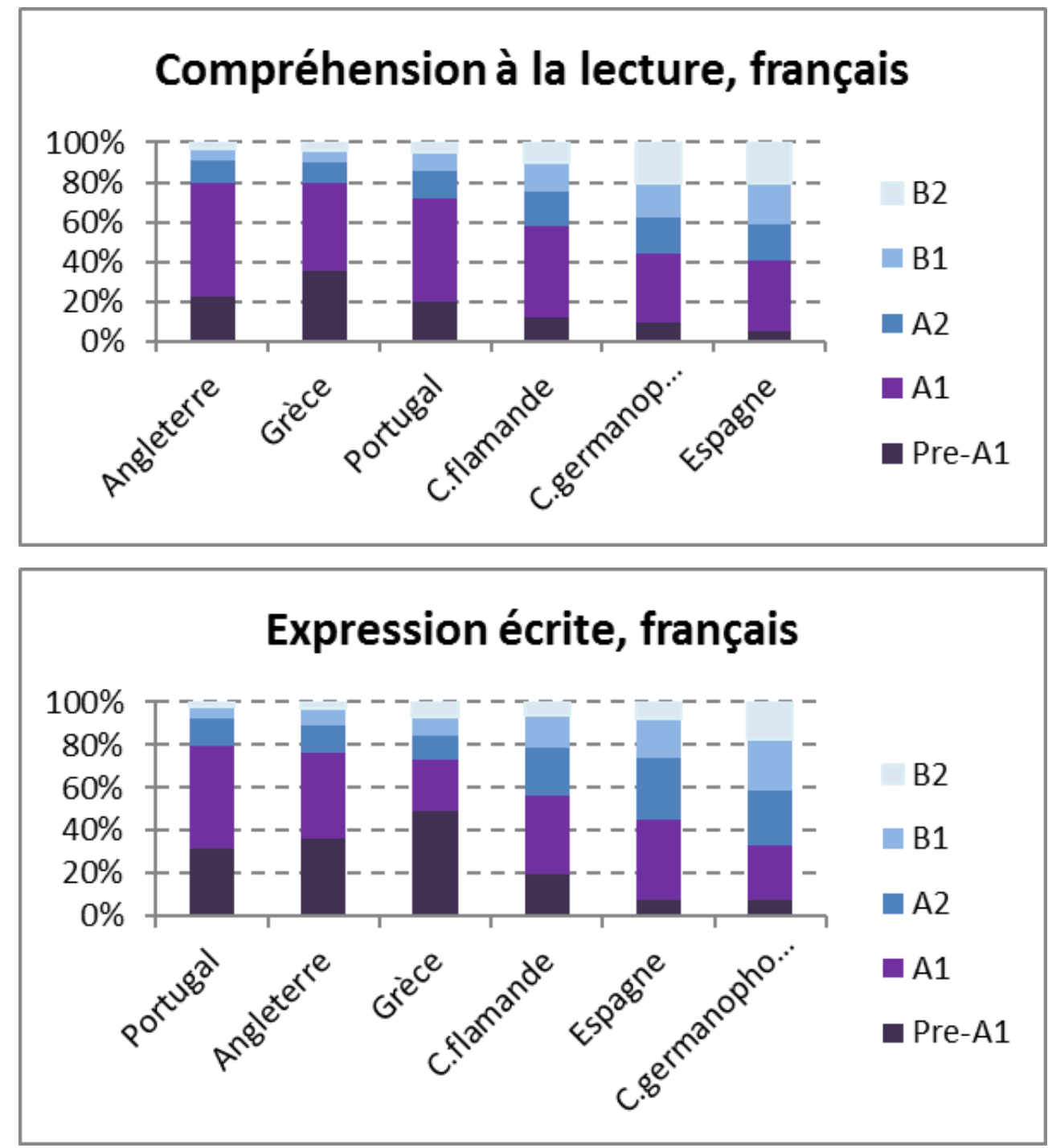

Les pays de langue romane ont des profils assez contrastés : alors que les élèves espagnols sont relativement nombreux aux niveaux de maitrise les plus avancé (respectivement $41 \%$ en compréhension à la lecture et $26 \%$ en expression écrite), les élèves portugais sont relativement peu nombreux à ces niveaux (respectivement 14 et $6 \%$ ). Les élèves de la Communauté germanophone de Belgique, qui bénéficient certainement de leur position géographique, sont près de $40 \%$ à atteindre le niveau d'indépendance pour les deux compétences. En Communauté flamande de Belgique, environ un quart des élèves atteignent les niveaux B1 et B2. La proportion d'élèves anglais pouvant être considérés comme autonomes est quant à elle très réduite (environ $10 \%$ dans les deux compétences. 


\section{Les liens entre les performances des élèves et le contexte d'apprentissage}

44 Le questionnaire de contexte destiné aux professeurs comportait notamment quatre questions sur la répartition du temps d'enseignement, l'importance de différents apprentissages, l'objet des travaux à domicile et les déterminants des notes finales attribuées à l'élève. Pour chacune de ces questions, les professeurs devaient se positionner par rapport aux huit propositions suivantes: compréhension à la lecture, compréhension à l'audition, culture et littérature, expression orale, grammaire, production écrite, prononciation et vocabulaire. Un indice composite a été calculé pour chacune des propositions, indice qui synthétise son importance relative dans les réponses d'un même professeur à chacune des quatre questions.

Des régressions marginales, qui examinent les relations entre les deux catégories de variables calculées indépendamment de toute autre influence, ont ensuite été calculées entre les indices de priorité des professeurs et les performances des élèves testés dans l'établissement où ils travaillent pour quatre compétences: la compréhension à la lecture, la compréhension à l'audition, la production écrite (facette communication) et la production écrite (facette correction). Sur l'ensemble des observations réalisées (16 systèmes éducatifs, 2 langues par système éducatif et 4 compétences, soit 128 observations), ces priorités relatives sont positivement liées aux performances dans deux tiers à trois quarts des observations, mais ces relations sont rarement significatives (au mieux, dans $11 \%$ des cas pour l'expression orale et $9 \%$ pour la culture ou la littérature et pour la grammaire).

Des analyses complémentaires ont été effectuées afin de dégager d'éventuelles relations entre les priorités des professeurs et leurs pratiques pédagogiques telles qu'elles apparaissent dans leurs réponses au questionnaire et les performances des élèves.

\section{Des analyses factorielles}

47 Des analyses factorielles ont été réalisées à propos de 17 indices constitués par SurveyLang sur la base des réponses des professeurs de langue en vue de les synthétiser en quelques facteurs davantage liés aux performances. Ces analyses ont porté sur l'ensemble des données recueillies, après exclusion des pays (ou communautés) dans lesquels le seuil de $50 \%$ de réponses n'avait pas été dépassé, le poids de chacun des pays restants étant rendu équivalent.

Les analyses aboutissent à un modèle qui distingue cinq facteurs, qui expliquent ensemble $59 \%$ de la variance des réponses.

49 Un premier facteur, qui explique à lui seul $25 \%$ de la variance, est appelé "Accent sur l'oral" car il est saturé ${ }^{7}$ par les variables suivantes :

- accent relatif sur l'expression orale ;

- accent relatif sur la compréhension à l'audition ;

- accent relatif sur une prononciation correcte ; 
- accent relatif sur le vocabulaire.

il est saturé par les variables suivantes :

- accent relatif sur la production écrite ;

- accent relatif sur la grammaire ;

- accent relatif sur la compréhension à la lecture ;

- accent relatif sur la culture et la littérature.

Un troisième facteur, qui explique $9 \%$ de la variance, est appelé "Exploitation des contacts" car il est saturé par les variables suivantes :

- fréquence d'utilisation de dispositifs de tics (technologies de l'information et de la communication) pendant l'enseignement ;

- création d'opportunités pour des échanges scolaires (visites);

- création d'opportunités pour des projets scolaires en langues.

Un quatrième facteur, qui explique $7 \%$ de la variance, est appelé "Exploitation de ressources spécifiques" car il est saturé par les variables suivantes :

- fréquence d'utilisation de contenus trouvés sur l'Internet pour l'enseignement ;

- utilisation du CECRL ;

- utilisation du portfolio des langues.

Enfin un cinquième facteur, qui explique $6 \%$ de la variance, est appelé "Apprentissage naturel"8 car il est saturé par les variables suivantes :

- langue cible parlée par les élèves ;

- langue cible parlée par le professeur;

- accent mis sur les similitudes entre la langue cible et d'autres langues (saturation négative).

\section{Des différences selon la langue cible}

Sur la base de ces facteurs, deux analyses complémentaires ont été réalisées: l'une aboutit à décrire la prépondérance respective de chacun de ces facteurs dans les différents systèmes éducatifs et à propos de chaque langue. L'autre examine les liens entre chacun de ces facteurs et les performances des élèves pour les différentes compétences.

La figure 11 présente la valeur moyenne de chacun des scores factorisés (avec son intervalle de confiance), en fonction de la langue cible.

L'analyse des scores moyens de l'ensemble des systèmes éducatifs dégage des profils différents selon la langue: selon les professeurs interrogés, l'enseignement de l'anglais fait largement appel à l'approche dite naturelle, tandis que dans le cas de l'allemand et du français, cette approche est peu prisée. Au-delà de ce point commun à l'allemand et au français, les professeurs de français et d'allemand mettent en œuvre des approches très différentes : dans le cas de l'allemand, l'oral et les contacts sont privilégiés, mais l'écrit est beaucoup moins présent; dans le cas du français, l'oral cède la place à l'écrit et l'exploitation des contacts n'est ni favorisée, ni rejetée. L'anglais occupe une position intermédiaire quant à l'accent mis sur l'oral ou sur l'écrit, mais se distingue nettement des deux autres langues en ce qui concerne l'exploitation des contacts (moins fréquente). 
Les valeurs du facteur d'exploitation de ressources spécifiques sont très faibles ou légèrement négatives (les professeurs de français tendent à les utiliser davantage que leurs collègues concernés par l'anglais).

Figure 11 - Valeurs moyennes et intervalles de confiance des 5 scores factorisés concernant les approches pédagogiques des professeurs, par langue cible

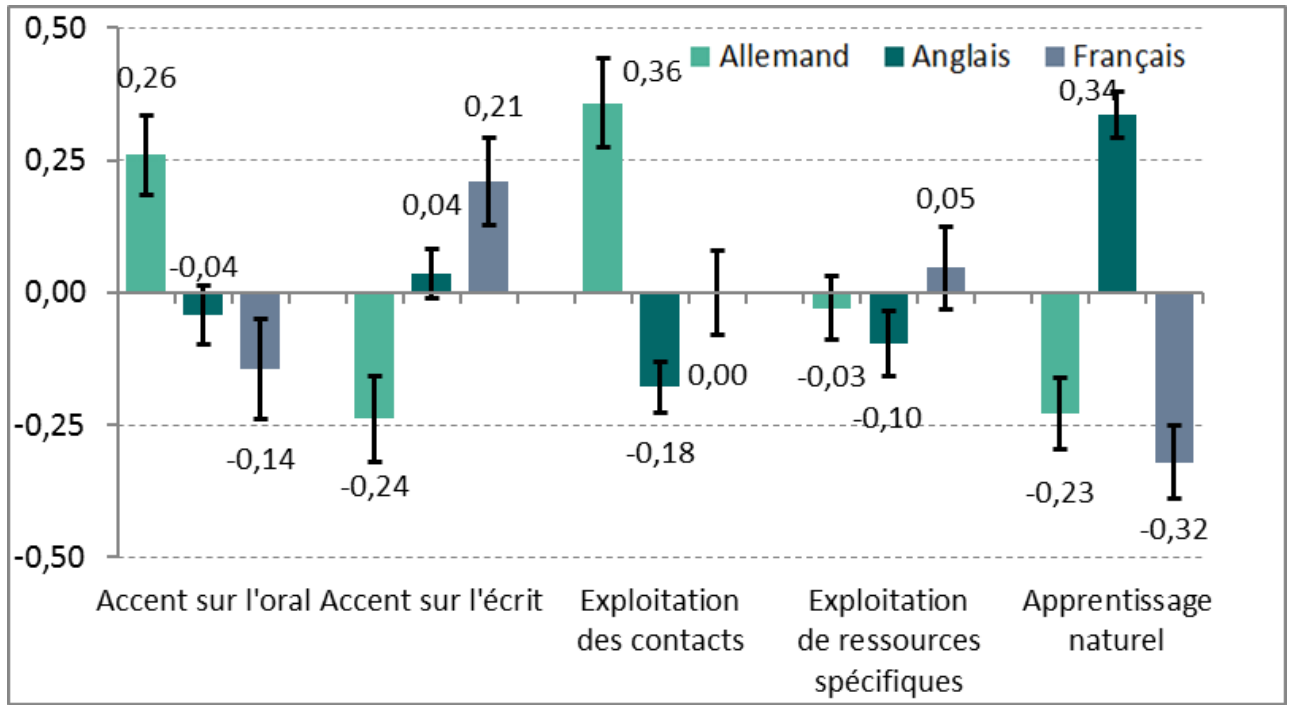

\section{Les approches mises en œuvre et les performances des élèves}

Des corrélations ont été calculées entre les scores factorisés décrits dans la section précédente et les performances des élèves en compréhension à la lecture et en expression écrite (sans distinguer les facettes communication et correction) dans l'ensemble des systèmes éducatifs (voir le tableau 2). Les corrélations sont faibles. Celles qui sont significatives sont en caractères gras.

59 Au plan européen, les résultats diffèrent selon la langue cible. C'est l'indice que nous avons appelé "approche naturelle" qui présente la relation la plus forte avec les performances pour les trois langues cibles ( 6 corrélations positives significatives, variant de 0,15 à 0,34 ).

Dans le cas de l'anglais, les cinq approches décrites à l'exception de l'exploitation de ressources spécifiques pour les deux compétences et de l'exploitation des contacts pour l'expression écrite sont corrélées aux performances des élèves. Si la tendance à mettre l'accent sur l'écrit, à exploiter les contacts et surtout à recourir à l'approche naturelle est associée à de meilleures performances, la tendance à mettre l'accent sur l'oral semble, quant à elle, contreproductive (corrélations négatives très faibles, mais significatives).

Tableau 2 - Corrélations entre les performances des élèves ( $L$ pour "compréhension à la Lecture" et E pour "Expression écrite") et les cinq indices (accent sur l'oral, accent sur l'écrit, exploitation des contacts, exploitation des ressources spécifiques et apprentissage naturel)

\begin{tabular}{|l|l|l|l|l|l|l|l|l|l|l|l|}
\hline & \multicolumn{3}{l}{ Oral } & \multicolumn{2}{l|}{ Écrit } & \multicolumn{3}{l|}{ Contacts } & \multicolumn{2}{l|}{ Ressources } & \multicolumn{2}{l|}{ Naturel } \\
\hline & L & E & L & E & L & E & L & E & L & E \\
\hline
\end{tabular}




\begin{tabular}{|l|c|c|c|c|c|c|c|c|c|c|}
\hline Allemand & $\mathbf{0 , 0 8}$ & $\mathbf{0 , 1 1}$ & 0,02 & 0,04 & $-0,02$ & 0,06 & $\mathbf{0 , 0 6}$ & 0,03 & $\mathbf{0 , 1 5}$ & $\mathbf{0 , 2 2}$ \\
\hline Anglais & $\mathbf{- 0 , 0 9}$ & $\mathbf{- 0 , 0 6}$ & $\mathbf{0 , 1 5}$ & $\mathbf{0 , 1 4}$ & $\mathbf{0 , 0 9}$ & 0,04 & $-0,01$ & $-0,04$ & $\mathbf{0 , 3 2}$ & $\mathbf{0 , 3 4}$ \\
\hline Français & 0,05 & 0,04 & $-0,08$ & $-0,09$ & 0,05 & 0,07 & $-\mathbf{0 , 1 4}$ & $-0,16$ & $\mathbf{0 , 2 9}$ & $\mathbf{0 , 3 4}$ \\
\hline
\end{tabular}

61 Par contre, dans le cas de l'allemand, c'est l'accent mis sur l'oral et l'approche naturelle qui sont positivement liés aux performances, ainsi que l'exploitation de ressources spécifiques, mais seulement dans le cas de la compréhension à la lecture. Bien que les corrélations soient dans l'ensemble plus basses que dans le cas de l'anglais, ce sont également les corrélations des performances avec l'approche naturelle qui présentent les valeurs les plus élevées (de 0,15 à 0,22 ).

Enfin dans le cas du français, on observe un contraste intéressant entre deux approches, qui s'esquissait déjà dans le cas de l'anglais : si le recours à l'approche naturelle s'avère, comme pour l'anglais et l'allemand, positivement corrélé aux résultats, l'exploitation de ressources spécifiques va de pair avec de moins bonnes performances.

\section{Discussion}

63 La faiblesse des corrélations entre les performances des élèves et les priorités des professeurs n'est pas surprenante. En effet, les professeurs interrogés dans chaque établissement scolaire sont ceux qui enseignent la langue cible au niveau de la CITE retenu et pas nécessairement ceux qui enseignent cette langue aux élèves testés. C'est sans doute la principale raison qui explique la faiblesse des liens entre les réponses données par les professeurs et les résultats des élèves, la force relative de ces liens étant notamment fonction de la cohérence entre les différents professeurs de l'établissement.

Sur l'ensemble des indices soumis à l'analyse factorielle, deux ont leur correspondant dans les réponses des élèves : un indice synthétise leur point de vue sur l'usage de la langue cible pendant les cours et l'autre la fréquence avec laquelle le professeur met l'accent sur les similitudes entre la langue cible et des langues connues. La corrélation entre les réponses des élèves et celles des professeurs est de 0,94 ou 0,83 en ce qui concerne l'utilisation de la langue cible (par le professeur ou par les élèves) et de 0,41 en ce qui concerne la fréquence avec laquelle le professeur fait remarquer les points communs entre la langue cible et d'autres langues lorsqu'il enseigne différents aspects de celle-ci. Ces deux pratiques pédagogiques, telles que décrites par les élèves, sont en moyenne, dans l'ensemble des systèmes éducatifs, liées aux performances des élèves : l'utilisation de la langue est liée positivement aux performances dans 82 observations sur 87 et 48 de ces relations sont significatives; l'accent sur les similitudes avec d'autres langues est, par contre, lié négativement aux résultats dans 74 cas sur 87,40 de ces relations négatives étant significatives. Malheureusement, l'ESLC ne permet pas de décrire plus finement les comportements que recouvre ce facteur: d'un côté, les didacticiens recommandent souvent d'appuyer la construction de nouvelles compétences sur ce qui est déjà maitrisé ; de l'autre, cependant, les références à "d'autres langues" pourraient correspondre à des similitudes inopportunes (comme l'explication du past perfect en anglais basée sur la grammaire française) ${ }^{9}$ ou à une maitrise insuffisante de la 
langue cible par le professeur, qui se traduirait par un recours fréquent à la langue d'enseignement.

Les réponses des élèves valident donc partiellement les analyses réalisées sur la base des réponses des professeurs.

Les différences d'approches efficaces selon la langue cible suggèrent quelques hypothèses. Tout d'abord les liens entre les indices regroupés sous l'intitulé "approche naturelle" peuvent-ils être considérés comme un argument en faveur de cette approche? Les liens négatifs de l'accent sur les similitudes entre langues rejoignent certainement ce que Krashen appelle "apprentissage" par opposition à l'acquisition ${ }^{10}$. Il faut cependant garder en mémoire qu'il s'agit ici d'un apprentissage lors de cours de langue qui comportent sans doute, dans la majorité des pays, une part importante d'enseignement : il pourrait être efficace de faire une plus grande place au "bain de langue", sans que cela signifie qu'il soit bénéfique d'exclure toute réflexion sur la langue, toute activité d'analyse. Les différences entre les approches liées aux résultats en fonction de la langue correspondent-elles à des caractéristiques des langues elles-mêmes, et/ou à leur place dans les représentations et dans l'environnement des élèves? L'ESLC a bien mis en évidence la place privilégiée de l'anglais de ces deux points de vue (voir SurveyLang, 2012 et Blondin, Goffin \& Baye, 2013). Peut-être une approche plus naturelle de la langue estelle plus efficace lorsque les élèves sont intéressés par son apprentissage, et lorsque cette langue est davantage présente dans leur environnement? Enfin les données analysées ne permettent pas d'exclure l'intervention d'un artéfact: les professeurs sont peut-être davantage enclins à utiliser la langue cible lorsqu'ils sont confrontés à des élèves plus compétents.

67 Sans doute sommes-nous ici face à une limite de l'enquête à large échelle: si cette approche a le mérite de mettre en évidence des phénomènes et de suggérer des pistes d'explication, elle soulève aussi des questions auxquelles elle ne peut pas répondre. Un travail plus clinique, des observations en classe et des recherches expérimentales complèteraient utilement l'étude.

\section{BIBLIOGRAPHIE}

Araùjo, L. \& Dinis da Costa, P. (2013). The European Survey on Language Competences: School-internal and External Factors in Language Learning. Luxembourg: Publications Office of the European Union.

Blondin, C. \& Demeuse, M. (2000). "Des indicateurs européens de qualité de l'éducation. Les langues étrangères : un premier exercice". Les Cahiers du Service de Pédagogie expérimentale, $\mathrm{n}^{\circ} 3-4$, pp. 161-170.

Blondin, C., Goffin, C. \& Baye, A. (2013). Les compétences en anglais et en allemand d'élèves de l'enseignement secondaire : participation de la Fédération Wallonie-Bruxelles à la Première étude européenne des compétences en langues. http://enseignement.be/index.php? page $=26044 \&$ id_fiche $=5461 \&$ dummy $=26379$, consulté le 13 juillet 2016 . 
Conseil de l'Europe (2001). Cadre européen commun de référence pour les langues : apprendre, enseigner, évaluer. Paris : Éditions Didier. http://www.coe.int/t/dg4/linguistic/Source/Framework_FR.pdf, consulté le 13 juillet 2016.

Dixon, L., Zhao, J., Shin, J.-Y., Wu, S., Su, J.-H., Burgess-Brigham, R., Unal Gezer, M. \& Snow, C. (2012). "What We Know About Second Language Acquisition: A Synthesis Form Four Perspectives". Review of Educational Research, volume 82, $\mathrm{n}^{\circ}$ 1, pp. 5-60.

EACEA/Eurydice (2012). Chiffres clés de l'enseignement des langues à l'école en Europe. Bruxelles: EACEA P9 Eurydice.

Krashen, S.D. (1985). The input hypothesis: issues and implications. New York : Longman.

Lafontaine, D. \& Blondin, C. (2004). Regards sur les acquis des élèves en Communauté française de Belgique. Apports des enquêtes de l'IEA, de Pisa et des évaluations externes. Bruxelles : De Boeck.

SurveyLang (2012). First European survey on language competences. Final report. Luxembourg : Office des publications de l'Union européenne. http://ec.europa.eu/languages/policy/strategicframework/documents/language-survey-final-report_en.pdf, consulté le 13 juillet 2016.

UNESCO (1997). Classification internationale type de l'éducation. Paris : UNESCO. http:// www.uis.unesco.org/Library/Documents/isced97-fr.pdf, consulté le 13 juillet 2016.

\section{NOTES}

1. La participation de la Communauté française à l'ESLC a bénéficié du soutien de la Fédération Wallonie-Bruxelles et elle a été assurée par l'Université de Liège (Services d'Analyse des systèmes et des pratiques d'enseignement et d'Approches quantitatives des faits d'enseignement).

2. Selon le Conseil de l'Europe, "Les compétences sont l'ensemble des connaissances, des habiletés et des dispositions qui permettent d'agir" (Conseil de l'Europe, 2001: 15). Nous utilisons le terme de "performance" pour désigner les activités ou comportements des élèves considérés comme des indicateurs de leurs compétences.

3. Pour rappel, les enquêtes à large échelle comportent généralement un essai de terrain (mise en œuvre des instruments d'enquête sur un échantillon réduit en vue de mettre à l'épreuve les instruments et les modalités de l'enquête), suivi d'une campagne principale, lors de laquelle sont collectées les informations qui feront l'objet des analyses et des publications.

4. Il s'agit d'une épreuve de lecture, sous la forme d'un test de closure ("texte à trous") : facile à administrer et à corriger, ce type de test donne à moindre cout un premier aperçu du niveau de compétence de l'élève.

5. Les compétences en espagnol et en italien n'ayant été évaluées respectivement que dans deux et un pays, ces deux langues n'ont pas été prises en compte pour cette analyse par langue.

6. Pour rappel, le mode est la valeur ou la modalité d'une variable le plus souvent observée. Une distribution est dite bimodale si deux valeurs ou modalités différentes ont des fréquences d'apparition à la fois proches et nettement plus élevées que les autres valeurs ou modalités.

7. "En analyse factorielle, la saturation d'une variable x par un facteur $F$ correspond à la corrélation entre $x$ (variable observée) et $F$ (variable latente). Elle quantifie le poids attribuable à $F$ dans la variance de $x . "$ (voir http://superieur.deboeck.com/resource/extra/9782804155421/PSYRAI_-_Glossaire.pdf).

8. L'expression "apprentissage naturel" fait référence à la théorie de Krashen (1985) pour qui l'acquisition d'une langue repose essentiellement sur les contacts avec cette langue (input hypothesis).

9. Merci à Germain Simons qui a suggéré cette explication. 
10. La distinction entre acquisition et apprentissage constitue la première des cinq hypothèses fondamentales de la théorie de Krashen: "There are two independent ways of developing abilities in second languages. 'Acquisition' is a subconscious process identical in all important ways to the process children utilize in acquiring their first language, while 'learning' is a conscious process that results in 'knowing about' language" (Krashen, 1985 : 77).

\section{RÉSUMÉS}

L'Étude européenne des compétences en langues ou ESLC (European Study of Language Competencies) a collecté des données sur les compétences en langues étrangères des élèves et sur les contextes d'apprentissage dans 16 systèmes éducatifs européens. Cette étude se base sur le Cadre européen commun de référence pour les langues (CECRL) : elle évalue les pourcentages d'élèves qui atteignent les différents niveaux du CECRL. La population visée est constituée des élèves d'une année de l'enseignement secondaire qui étudient la langue cible depuis au moins une année scolaire complète. Cette contribution présente les résultats de l'étude en ce qui concerne la compréhension à la lecture et l'expression écrite en allemand, anglais et français. Les réponses des professeurs à un questionnaire permettent de décrire certaines de leurs pratiques, qui diffèrent selon la langue concernée, tout comme leurs liens avec les performances des élèves.

The European Survey on Language Competences or ESLC collected data on students' foreign language competences and learning environments in 16 European education systems. This study is based on the Common European Framework of Reference for Languages (CEFR): it assesses the percentage of students who reach the different levels of the CEFR. The target population consists of students from one grade of secondary education who study the target language for at least one full school year. The paper presents the results of the study with regard to reading comprehension and writing in German, English and French. The responses of the teachers to a questionnaire make it possible to describe some of their practices, which differ according to the language concerned, just like their links with the performances of the students.

\section{INDEX}

Mots-clés : langue étrangère, lecture, écriture, enseignement secondaire, évaluation, compétence, CECRL, ESLC, priorités, pratiques pédagogiques

Keywords : foreign language, reading, writing, secondary education, assessment, competence, CEFRL, ESLC, priorities, teaching practices

\section{AUTEURS}

\section{CHRISTIANE BLONDIN}

CHRISTELLE GOFFIN

ARIANE BAYE 